\title{
Central vein rupture during percutaneous transluminal angioplasty for central vein stenosis or occlusion in haemodialysis patients
}

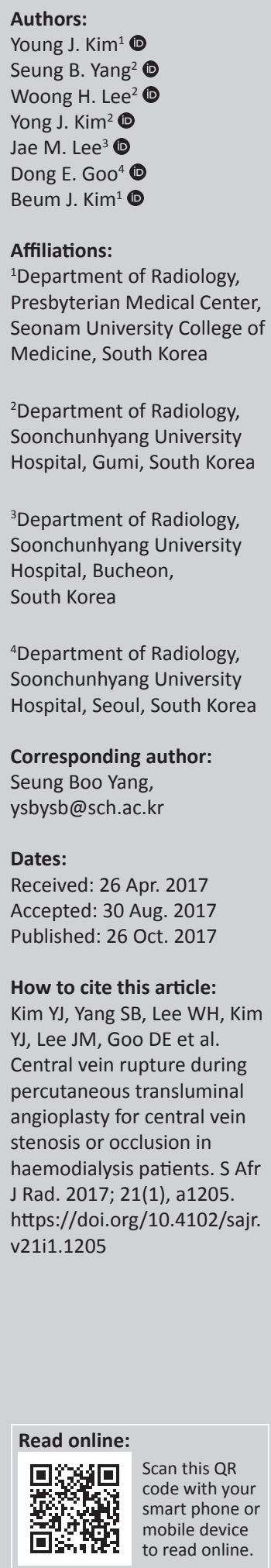

Background: Endovascular treatments such as percutaneous transluminal angioplasty (PTA) and stent placements are becoming the standard method in managing haemodialysis access failure. Venous rupture is the most common complication during endovascular procedures. Complications during endovascular treatments of central venous stenosis or occlusion have been rarely reported.

Objectives: To investigate the incidence and management of central vein rupture while performing PTA for central vein stenosis or occlusion in haemodialysis patients.

Method: Between 1998 and 2013,PTA was performed using various techniques in haemodialysis patients for central vein stenoses $(n=2437)$ and occlusions $(n=666)$. When the guide wire passed through the stenosis or the occlusion, PTA was performed regardless of the presence of a venous rupture. The incidence of central vein rupture was analysed using the chi-square test according to the gender, location, right versus left, presence of thrombosis and stenosis versus occlusion. Percutaneous management of central vein rupture was also evaluated.

Results: Central vein rupture occurred in 12 cases (0.39\%). All ruptures occurred in the cases with occlusion. Only stenosis versus occlusion reflected a significant correlation $(p<0.001)$ with central vein rupture. Central vein ruptures were managed by low-pressure balloon tamponade $(n=2)$, stent/stent-graft $(n=5)$ and balloon-mediated haemostasis blocking venous inflow followed by the observation $(n=5)$.

Conclusion: Central vein rupture is a rarely occurring complication while performing PTA for central vein stenosis and occlusion, and the majority can be successfully managed by percutaneous techniques.

\section{Introduction}

Haemodialysis access failure is often the most frequent cause of hospitalisation in patients with end-stage renal disease (ESRD) requiring haemodialysis. ${ }^{1}$ The common causes of access failure are venous stenosis and thrombosis. ${ }^{2}$ Endovascular treatments such as percutaneous transluminal angioplasty (PTA) and stent placement are becoming the standard method in managing these problems. ${ }^{2}$ During the endovascular procedure, multiple complications can arise such as venous or venous anastomotic rupture, arterial emboli and venous dissections. ${ }^{2}$ Among these complications, venous rupture is the most common complication with a reported incidence of $0.7 \%-4.6 \% .^{2,3,4,5}$

The incidence of central venous stenosis or occlusion is reported at $11 \%-50 \% 0^{6,7,8}$ and when this occurs ipsilateral to the functioning haemodialysis access, it can ultimately cause access failure. PTA and stent placement are considered as effective primary treatment options in treating central venous stenosis or occlusion., 9,10,11,12 However, there have been very few case reports about complications that may arise while performing PTA and stent placement for central venous stenosis or occlusion. ${ }^{13}$

The purpose of this study is to investigate the incidence and management of central vein rupture that can occur while performing PTA for central vein stenosis or occlusion in the haemodialysis patient.

\section{Research method and design}

A retrospective, analytic, cross-sectional study design was used to investigate the incidence and management of central vein rupture during PTA for central vein stenosis or occlusion in the 
haemodialysis patient. The institutional review board approved this retrospective study; the need for informed consent was waived.

\section{Patients}

The study included 3103 haemodialysis patients who presented between March 1998 and December 2013 with central vein stenosis or occlusion and required PTA. We reviewed these patients' medical records and imaging on the picture archiving and communication system (PACS) to obtain data.

\section{Procedure}

An 8-Fr vascular sheath (Terumo, Tokyo, Japan) was inserted into the fistula, graft or vein proximal to the fistula or graft. On venography, the presence or absence of contrast media beyond the stenotic lesion defined stenosis or occlusion, respectively. When a steno-occlusive lesion was demonstrated on the venogram, passage of a 0.035 -inch guide wire (Terumo, Tokyo, Japan) and angiographic catheter (5-Fr Kumpe catheter, Cook, Bloomington, IN, USA) through the steno-occlusive lesion was attempted. If passage through the steno-occlusive lesion was successful, PTA was performed with the use of a $12 \mathrm{~mm}-$ $14 \mathrm{~mm}$ balloon (Conquest or Atlas, C. R. Bard, Covington, GA, USA). If passage through the steno-occlusive lesion failed, recanalisation from the opposite end of the stenosis, from central aspect (i.e. distal site of the steno-occlusive lesion), via the right femoral vein was attempted. If this failed, the reverse tip of a 0.035-inch guide wire was used for recanalisation. When all of these failed and if there was no venous rupture, then the Colapinto needle (Cook, Bloomington, IN, USA) was used for recanalisation (Figure 1). The Colapinto introducer sheath (Cook, Bloomington, IN, USA) was inserted in the venous limb of the arteriovenous fistula and advanced in the antegrade direction with venous flow to the steno-occlusive lesion. A diagnostic catheter inserted in the right femoral vein was advanced to the portion distal to the steno-occlusive lesion. Under fluoroscopic guidance, the trocar stylet was advanced via the Colapinto sheath towards the diagnostic catheter distal to the steno-occlusive lesion. ${ }^{14}$

Central vein rupture was defined as extravasation of contrast media observed on the venogram. In the presence of central vein rupture, if the wire was already through the stenotic lesion, the balloon (Conquest or Atlas, C. R. Bard, Covington, GA, USA) was inflated with low pressure (2 $\mathrm{mmHg}-3$ $\mathrm{mmHg})$. If this balloon tamponade failed, a stent or stentgraft was placed (Figure 2).

When the guide wire failed to pass through the rupture site, the balloon was inflated proximal to the rupture site to block

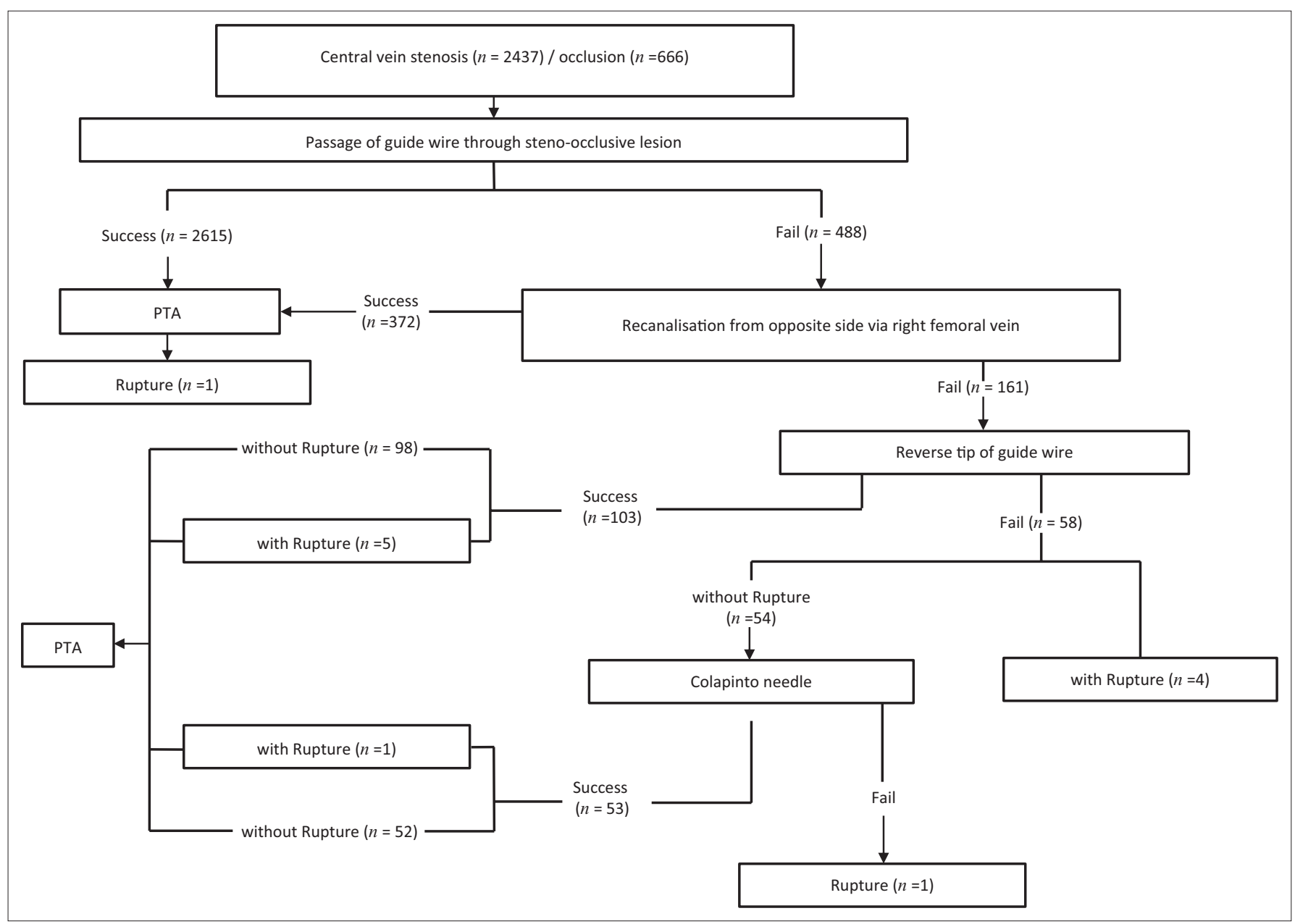

FIGURE 1: Flowchart of the percutaneous transluminal angioplasty for central venous stenosis and occlusion. 


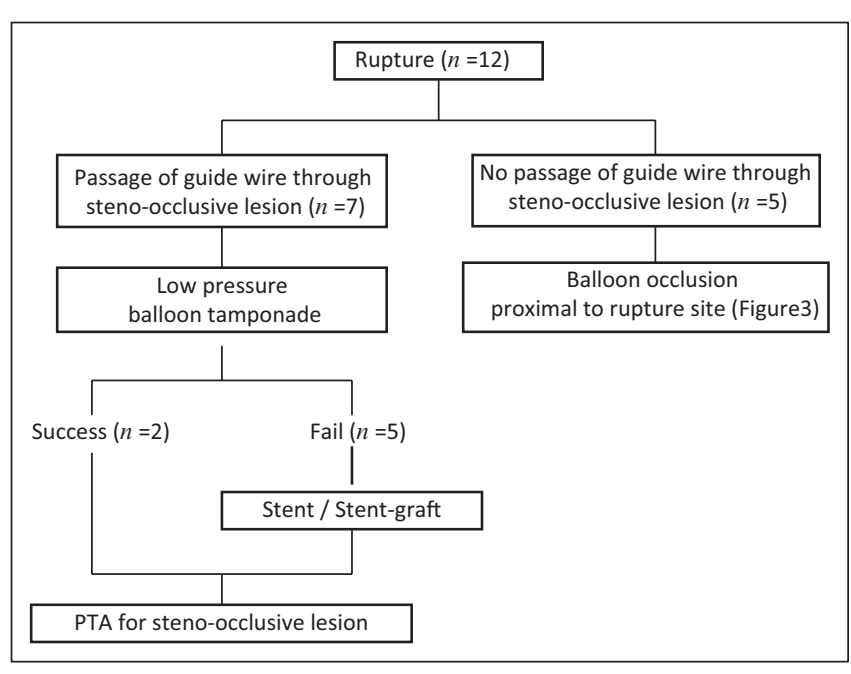

FIGURE 2: Flowchart of the management of central vein rupture while performing percutaneous transluminal angioplasty.

the venous inflow (Conquest or Atlas, C. R. Bard, Covington, GA, USA) and this was followed by observation (Figure 3).

\section{Analyses}

The chi-square test was used to evaluate the incidence of central vein rupture according to the location (subclavian versus innominate vein), gender, right versus left, thrombosis and stenosis versus occlusion.

\section{Results}

From March 1998 to December 2013, PTA for central vein stenosis $(n=2437)$ or occlusion $(n=666)$ was performed in 3103 haemodialysis patients (mean age, $59.5 \pm 12.1$ years; range, 20-91 years). The central venous stenosis or occlusion was located in the subclavian $(n=1368)$ and the innominate $(n=1735)$ veins. The number of right- and left-sided central venous stenosis or occlusion was 1287 and 1816, respectively. Of these, 103 cases accompanied venous thrombosis. The study group consisted of 1445 men (mean age, $58.8 \pm 12.8$ years; range, 22-90 years) and 1658 women (mean age, $60.1 \pm$ 11.9 years; range, 20-91 years).

Twelve cases of central vein rupture occurred during intervention $(0.39 \%)$. These were during balloon dilatation $(n=1)$, recanalisation using the reverse tip of the guide-wire $(n=9)$ or use of the Colapinto needle $(n=2)$. All of the central vein ruptures occurred in cases with occlusion rather than stenosis. The central vein ruptures were located in the innominate $(n=9)$ and the subclavian $(n=3)$ veins. Among the evaluated factors, location $(p=0.409)$, gender $(p=0.811)$, thrombosis $(p=0.331)$ and right versus left $(p=0.081)$ did not have significant correlation with central vein rupture while performing PTA. Only stenosis versus occlusion factor $(p<0.001)$ showed significant correlation with central vein rupture while performing PTA.

In seven cases of venous rupture, the guide wire was successfully mediated through the steno-occlusive lesion ( $n=7,58.33 \%)$. Of these, two cases were successfully managed with low-pressure balloon tamponade. In the five cases where balloon tamponade failed, a self-expandable stent or stent-graft was used for successful haemostasis.

In the five cases where the guide wire could not pass through the steno-occlusive lesion $(n=5,41.67 \%)$, PTA could not be performed. In these patients, balloon inflation was used to induce occlusion proximal to the rupture site, blocking the venous inflow and was followed by observation (Figure 3). Using this technique, no leakage of contrast media at the rupture site was observed on the venogram in all of the five cases.

The success rate of PTA in managing central venous stenosis or occlusion was $99.84 \%$. In the case of central vein rupture, the success rate of PTA was $58.33 \%$.

\section{Discussion}

Central venous stenosis or occlusion ipsilateral to the functioning haemodialysis access may render it dysfunctional. ${ }^{6,7}$ The incidence of central venous stenosis or occlusion is reported at $11 \%-50 \%{ }^{6,7,8}$ Steve et al. ${ }^{8}$ report that the prevalence of subclavian vein stenosis is about $11 \%$ and the predisposing factors are previous history of ipsilateral subclavian vein cannulation and old aged ipsilateral arteriovenous fistula. They proposed fibrosis nidus formation because of subclavian cannulation and increased blood flow because of arteriovenous fistula as the mechanism of central venous stenosis.

Several studies have reported on the use of PTA in the management of central venous stenosis..$^{8,11,15,16}$ Treating central venous lesions with PTA resulted in a higher and earlier recurrence rate compared with PTA for peripheral lesions. ${ }^{11,15}$ Intravascular ultrasound (IVUS) of the central venous lesions post-PTA balloon dilatation exhibited severe elastic recoil, which explains the higher and earlier recurrence rate of the central venous lesions. ${ }^{16}$ The management of central venous lesions with stents instead of PTA showed better results and outcome. ${ }^{7,11,12}$

Kim et al. $^{2}$ reported on procedure-related complications during recanalisation of haemodialysis access. Venous/ venous anastomotic rupture, arterial emboli, venous/intragraft dissection, graft extravasation, haematoma at the puncture site, arterial rupture, balloon fragmentation and symptomatic pulmonary embolism, all are the complications that could arise during the recanalisation. Among these complications, venous/venous anastomotic rupture was the most common (4.6\%) complication, and in most cases $(98.7 \%)$, these were managed successfully with the use of balloon tamponade and stents. Rates of venous rupture during angioplasty of haemodialysis access are reported to range between $0.7 \%$ and $4.6 \% .^{3,4,5}$ Various methods such as observation, balloon tamponade, stent or stent-graft were used in treating venous rupture, and they all indicated favourable outcomes. ${ }^{3,13,17,18,19,20,21}$ In the case of using a bare stent, although bleeding through interstices of the stent can 

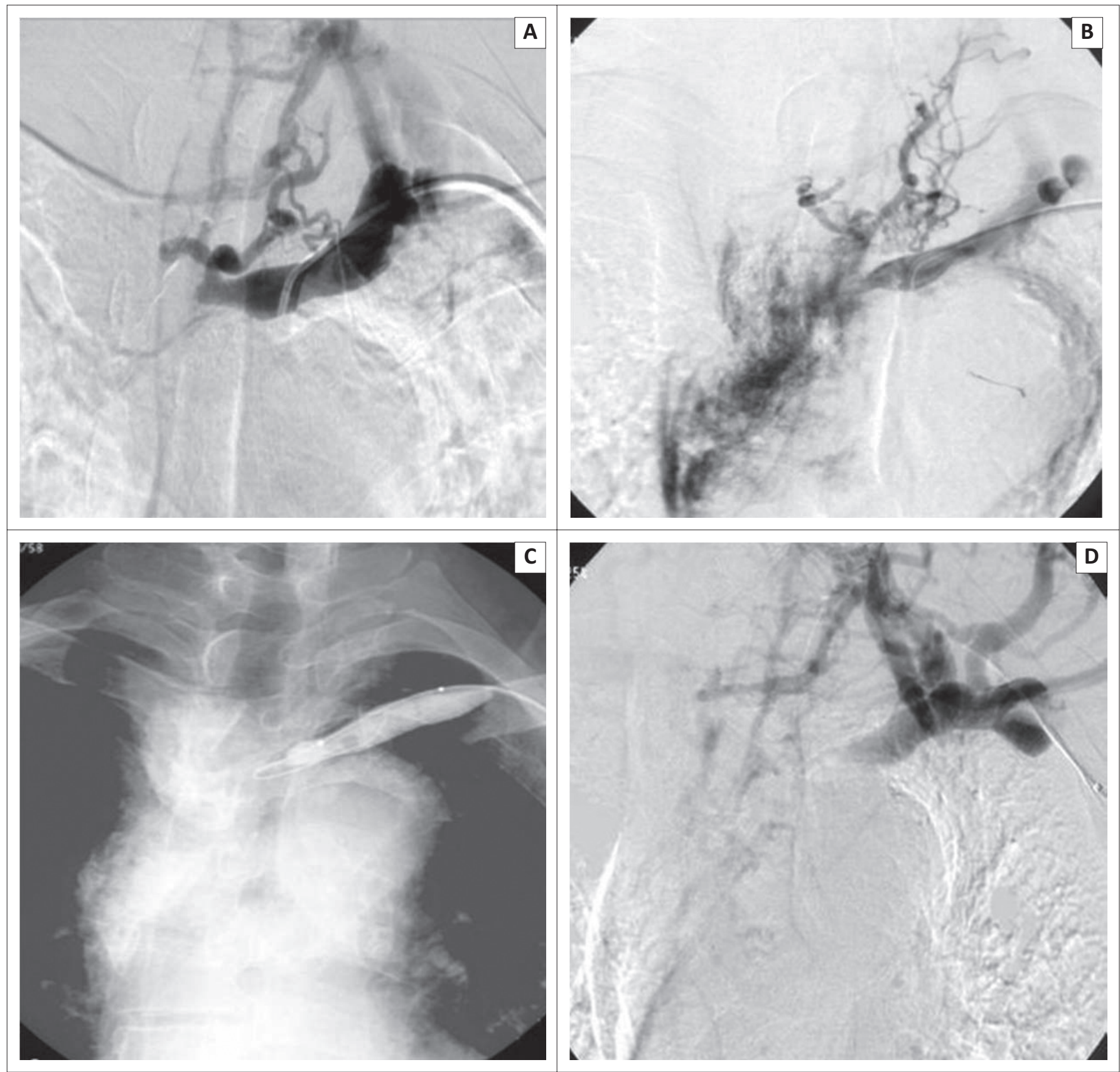

FIGURE 3: A 54-year-old man. Left radio-cephalic fistula. Fistulogram: (a) complete obstruction in the left innominate vein; (b) massive contrast leakage into the mediastinal space because of a guide wire induced innominate vein rupture; (c) balloon occlusion proximal to the rupture site was performed during 10 min, because of failure of guide wire passage through the rupture site; (d) absence of contrast extravasation on the final fistulogram.

be suspected, these bleedings are very minimal and the use of the bare stent was effective in the management of venous rupture bleeding. Stent deployment is hypothesised to be effective and safe by providing a low-resistance conduit for blood flow in treating venous rupture. . $^{3,17,18,19,20,21}$

There have only been a few case reports about the complications that can arise during the interventional management of central venous stenosis or occlusion, particularly with regards to venous rupture..$^{2,22,23}$ In this study, the incidence of central vein rupture was very rare $(0.39 \%)$ and occurred only with central venous occlusion. Techniques such as balloon tamponade $(n=2)$, stent or stent-graft $(n=5)$ and balloon occlusion proximal to the rupture site followed by observation ( $n=5$ ) were used to subsequently achieve successful haemostasis.

Rebecca et al. ${ }^{24}$ reported more rapid stenosis progression and escalation of lesions associated with the PTA treatment of high-grade $(>50 \%)$ asymptomatic central vein stenosis, compared to the non-treatment groups. They suggested that the PTA procedures are not always beneficial (12\% and $88 \%$ of regression and progression rate, respectively) and in some instances, can be detrimental (escalation of central venous stenosis in $8 \%$ of treated group) especially in the cases of asymptomatic central vein stenosis that does not present with swelling in the arm, breast or face and haemodialysis failure. However, untreated central vein stenosis could eventually 
develop thrombosis and occlusion. According to our study, occlusion seems to be an important risk factor for central vein rupture and can lengthen the total interventional procedure time. In $40 \%$ of central vein rupture cases, guide wires failed to pass through the steno-occlusive lesion making PTA impossible to perform. In other words, haemodialysis access becomes unavailable and a new haemodialysis access route will be required. In this aspect, early PTA management of central vein stenosis detected on the venography or fistulography may be a better method in maintaining the haemodialysis access route and, thus, maybe more safe and useful.

\section{Limitations of the study}

Limitation of this study was the retrospective design and single centre study which did not allow extensive statistical analysis.

\section{Conclusion}

In conclusion, central vein rupture occurs very rarely while performing PTA for central vein stenosis and occlusion, and the majority are easily manageable by percutaneous techniques. In the setting of free passage of guide wire through the steno-occlusive lesion, PTA can be performed successfully to manage central venous stenosis or occlusion even in the presence of central vein rupture.

\section{Ethical consideration}

The institutional review board approved this retrospective study; the need for informed consent was waived. No ethical clearance number was provided.

\section{Acknowledgements}

This study received funding from Soonchunhyang University Industry Academy Cooperation Foundation.

\section{Competing interests}

The authors declare that they have no financial or personal relationship(s) that may have inappropriately influenced them in writing this article.

\section{Authors' contributions}

S.B.Y. and Y.J.K. were guarantors of integrity of the entire study. D.E.G. did statistical analysis. All authors were responsible for study concepts, study design, data acquisition, data analysis and data interpretation; manuscript drafting or manuscript revision for important intellectual content; manuscript final version approval; literature research; clinical studies; and manuscript editing.

\section{References}

1. Mayers JD, Markell MS, Cohen LS, et al. Vascular access for hemodialysis: Variable in hospital stay. ASAIO J. 1992;38:113-115.

2. Kim DH, Goo DE, Yang SB, et al. Endovascular management of immediate procedure-Related complications of failed hemodialysis access recanalization. Korean J Radiol. 2005;6:185-195. https://doi. org/10.3348/kjr.2005.6.3.185

3. Bittl JA. Venous rupture during percutaneous treatment of hemodialysis fistulas and grafts. Catheter Cardiovasc Interv. 2009;74:1097-1011. https://doi.org/ 10.1002/ccd.22143

4. Bathard GA. Percutaneous transvenous angioplasty in the treatment of vascular stenosis. Kidney Int. 1992;42:1390-1397. https://doi.org/10.1038/ ki.1992.431

5. Turmel-Rodriguez L, Pengloan J, Blanchier D, et al. Insufficient dialysis shunts: Improved long term patency rates with close hemodynamic monitoring, repeated balloon angioplasty, and stent placement. Radiology 1993;187:273-278. https:// doi.org/10.1148/radiology.187.1.8451428

6. Hernandez D, Diaz F, Rufino $M$, et al. Subclavian vascular access stenosis in dialysis patients: Natural history and risk factors. J Am Soc Nephrol. 1998;9:1507-1510.

7. Haage $P$, Vorwerk $D$, Piroth $W$, et al. Treatment of hemodialysis-related central venous stenosis or occlusion: Results of primary wall stent placement and followup in 50 patients. Radiology 1999;212:175-180. https://doi.org/10.1148/ radiology.212.1.r99j|21175

8. Schwab SJ, Quarles LD, Middletone JP, et al. Hemodialysis-associated subclavian vein stenosis. Kidney Int. 1988;33:1156-1159. https://doi.org/10.1038/ki. 1988.124

9. NKF-K/DOQI clinical practice guidelines for vascular access. National kidney foundation-Dialysis outcomes quality initiative. Am J Kidney Dis. 1997;30 (4 suppl 3):S150-S191.

10. NKF-K/DOQI clinical practice guidelines for vascular access: Update 2000. Am Kidney Dis. 2001;37(1 suppl 1):S137-S181. https://doi.org/10.1016/S0272 6386(01)70007-8

11. Kovalik EC, Newman GE, Suhocki P, et al. Correction of central venous stenoses: Use of angioplasty and vascular Wallstents. Kidney Int. 1994;45:1177-1181. https://doi.org/10.1038/ki.1994.156

12. Shoenfeld R, Hermans $H$, Novick A, et al. Stenting of proximal venous obstructions to maintain hemodialysis access. J Vasc Surg. 1994;19:532-538. https://doi. org/10.1016/S0741-5214(94)70082-6

13. Valentin CN, Zangan SM. Axillary vein rupture after angioplasty. Semin Intervent Radiol. 2009;26:276-8. https://doi.org/10.1055/s-0029-1225673

14. Goo DE, Kim YJ, Choi DL, et al. Use of a Rosch-Uchida needle for recanalization of refractory dialysis-Related central vein occlusion. AJR 2010;194:1352-1356. https://doi.org/10.2214/AJR.09.3485

15. Newman GE, Davidson CJ, McCann RL, et al. Functional restenosis after hemodialysis graft angioplasty (abstr). J Am Soc Nephrol. 1991;2:341.

16. Davidson CJ, Newman GE, Sheikh KH, et al. Mechanism of angioplasty in hemodialysis fistula stenoses evaluated by intravascularr ultrasound. Kidney Int. 1991;40:91-95. https://doi.org/10.1038/ki.1991.185

17. Rundback JH, Leonardo RF, Poplausky MR, et al. Venous rupture complicating hemodialysis access angioplasty: Percutaneous treatment and outcomes in seven patients. Am J Roentgenol. 1998;171:1081-1084. https://doi.org/10.2214/ajr. 171.4.9763001

18. Rajan DK, Clark TW. Patency of wall stents placed at the venous anastomosis of dialysis grafts for salvage of angioplasty-Induced rupture. Cardiovasc Intervent Radiol. 2003;26:242-245. https://doi.org/10.1007/s00270-003-2706-x

19. Funaki B, Szymski GX, Leef JA, et al. Wall stent deployment to salvage dialysis graft thrombolysis complicated by venous rupture: Early and intermediate results. Am J Roentgenol. 1997;169:1435-1437. https://doi.org/10.2214/ajr.169.5.9353476

20. Raynaud AC, Angel CY, Sapoval MR, et al. Treatment of hemodialysis access rupture during PTA with Wall stent implantation. J Vasc Interv Radiol. 1998;9: 437-442. https://doi.org/10.1016/S1051-0443(98)70295-7

21. Patel RI, Peck SH, Cooper SG, et al. Patency of wall stents placed across the venous anastomosis of hemodialysis grafts after percutaneous recanalization. Radiology 1987;209:365-370. https://doi.org/10.1148/radiology.209.2.9807560

22. Weng FL, Berns JS. Complications of percutaneous treatment of thrombosed hemodialysis access grafts. Semin Dial. 2003;16:257-262.

23. Sofocleous SO, Schur I, Koh E, et al. Percutaneous treatment of complications occurring during hemodialysis graft recanlization. Eur J Radiol. 2003;47:237-246. https://doi.org/10.1016/S0720-048X(02)00087-6

24. Levit RD, Cohen RM, Kwak A, et al. Asymptomatic central venous stenosis in hemodialysis patients. 